\title{
Moderate Galaxy-Galaxy Lensing
}

\author{
Shude Mao ${ }^{1,2 \star}$, Jian Wang ${ }^{3}$, Martin C. Smith ${ }^{1,4}$ \\ ${ }^{1}$ National Astronomical Observatories, Chinese Academy of Sciences, 20A Datun Road, Beijing 100012, China \\ ${ }^{2}$ Jodrell Bank Centre for Astrophysics, the University of Manchester, Manchester, M13 9PL, UK \\ ${ }^{3}$ Department of Physics and Tsinghua Center for Astrophysics, Tsinghua University, Beijing 100084, China \\ ${ }^{4}$ The Kavli Institute for Astronomy and Astrophysics, Peking University, Yi He Yuan Lu 5, Beijing 100871, China
}

Accepted .........Received .......; in original form .......

\begin{abstract}
We study moderate gravitational lensing where a background galaxy is magnified substantially, but not multiply imaged, by an intervening galaxy. We focus on the case where both the lens and source are elliptical galaxies. The signatures of moderate lensing include isophotal distortions and systematic shifts in the fundamental plane and Kormendy relation, which can potentially be used to statistically determine the galaxy mass profiles. These effects are illustrated using Monte Carlo simulations of galaxy pairs where the foreground galaxy is modelled as a singular isothermal sphere model and observational parameters appropriate for the Large Synoptic Survey Telescope (LSST). The range in radius probed by moderate lensing will be larger than that by strong lensing, and is in the interesting regime where the density slope may be changing.
\end{abstract}

Key words: Gravitational lensing - galaxies: elliptical and lenticular - dark matter - Galaxies: structure, Galaxies: formation

\section{INTRODUCTION}

Gravitational lensing is usually divided into microlensing, strong lensing, and weak gravitational lensing. Microlensing refers to the temporal change of magnification of a background source lensed by an intervening object (usually stars). Strong lensing occurs when multiple images or strong distortions (e.g. giant arcs) of a background source are caused by an intervening galaxy or cluster. For weak lensing the distortion of a background source by the lensing object is much more subtle. For example, a circular source will be lensed into an ellipse with ellipticity of a few percent. Such subtle deviations have to be inferred statistically by averaging over a large number of background galaxies. All these fields in gravitational lensing have been extensively studied, with diverse applications ranging from cosmology to the detection of extrasolar planets (for a review on these topics, see Schneider et al. 2006 and references therein).

In this work, we shall explore the intermediate regime which we term as "moderate gravitational lensing". In this case, the magnification is still significant, but no multiple images occur. In the context of clusters of galaxies, Williams \& Lewis (1998) and Futamase et al. (1998) have considered a class of gravitationally lensed, highly magnified, yet morphologically regular images (originally motivated by observations of the object cB58, Yee et al. 1996, Seitz et al. 1998). More recently, Sonnenfeld et al. (2011) discussed the magnification effect in with a focus on how to remove the masssheet degeneracy. In this paper, we shall explore the case where an elliptical galaxy is lensed by a foreground elliptical galaxy. Many cases are expected to be found in future surveys (e.g. by PANSTARRS ${ }^{1}$ and LSST ${ }^{2}$, where hundreds of millions of galaxies will be imaged. Many pairs of galaxies that are close to each other but at different redshifts will be discovered. Most of these will not be multiply-imaged. At very large separations, a weak-lensing galaxy-galaxy analysis will be appropriate; at very small separations, multiple images may form. In this paper we carry out Monte Carlo simulations of moderate galaxy-galaxy lensing at intermediate separations to illustrate the signatures, and what we can learn from these.

The paper is organised as follows. In Section 2 we describe the simple singular isothermal sphere model we use. In Section 3. we present the main results, including the predictions for the optical depth, isophotal distortions, and systematic offsets in the fundamental plane and Kormendy relation. We present a summary and discussion in Section 4 . Throughout this paper, we adopt a flat $\Lambda \mathrm{CDM}$ cosmology model with $\Omega_{\mathrm{m}, 0}=0.27$ and $\Omega_{\Lambda, 0}=0.73$ for the matter and cosmological constant, and the Hubble constant is written as $H_{0}=100 h \mathrm{~km} \mathrm{~s}^{-1} \mathrm{Mpc}^{-1}$ with $h=0.705$ Komatsu et al. 2009).

\footnotetext{
^ shude.mao@gmail.com; jian-w06@mails.tsinghua.edu.cn
}

\footnotetext{
1 http://pan-starrs.ifa.hawaii.edu/public/home.html

2 http://www.lsst.org
} 


\section{THE LENS AND SOURCE MODEL}

In this section, we describe the lens mass model, the source population and the surface brightness models of foreground and background galaxies which we will use for Monte Carlo simulations of galaxy pair catalogues.

\subsection{Lens mass model}

For simplicity, we model the mass profile of the lensing galaxy as a singular isothermal sphere (SIS). This simple model is analytically tractable, and fits the observational data well (e.g., Koopmans 2009). The surface mass density can be modelled as

$\Sigma(\xi)=\frac{\sigma^{2}}{2 G} \frac{1}{\xi}$,

where $\xi$ is the (physical) distance in the lens plane, and $\sigma$ is the one-dimensional velocity dispersion. The angular Einstein radius of an SIS is given by

$\theta_{\mathrm{E}}=4 \pi \frac{\sigma^{2}}{c^{2}} \frac{D_{\mathrm{ds}}}{D_{\mathrm{s}}}=1 . " 4\left(\frac{\sigma}{220 \mathrm{~km} \mathrm{~s}^{-1}}\right)^{2} \frac{D_{\mathrm{ds}}}{D_{\mathrm{s}}}$,

where $c$ is the speed of light, $D_{\mathrm{ds}}$ is the angular diameter distance between the lens and the source, and $D_{\mathrm{s}}$ is the angular diameter distance to the source.

A point source at unlensed angular position $0<\theta_{\mathrm{s}}<\theta_{\mathrm{E}}$ from the galaxy produces two images at the angular positions $\theta_{1,2}=\theta_{\mathrm{s}} \pm \theta_{\mathrm{E}}$. The magnification of each image and the total absolute magnification are given by

$\mu_{1,2}=\frac{\theta_{1,2}}{\theta_{\mathrm{s}}}, \quad \mu_{\text {total }}=\left|\mu_{1}\right|+\left|\mu_{2}\right|=2 \frac{\theta_{\mathrm{E}}}{\theta_{\mathrm{s}}}, \quad 0<\theta_{\mathrm{s}}<\theta_{\mathrm{E}}$.

Notice that the total magnification for the two images exceeds two.

A point source at angular separation $\theta_{\mathrm{s}}>\theta_{\mathrm{E}}$ produces only one image at the angular position $\theta=\theta_{\mathrm{s}}+\theta_{\mathrm{E}}$, with magnification $\left(\theta_{\mathrm{E}}+\theta_{\mathrm{s}}\right) / \theta_{\mathrm{s}}$, giving a value between 1 and 2 . Thus if a source is a few Einstein radii away from the line of sight, it may still experience substantial magnification and differential magnification, and have observable signatures.

\subsection{Lensing probability}

For a background elliptical galaxy at redshift $z_{\mathrm{s}}$, the lensing probability (optical depth) can be obtained as follows

$\tau\left(z_{\mathrm{s}}\right)=\int_{0}^{z_{i}} d z_{\mathrm{d}} \int d \sigma \Phi_{\sigma}\left(z_{\mathrm{d}}, \sigma\right) \sigma_{\mathrm{cr}}(\sigma) \frac{c d t}{d z_{\mathrm{d}}}$

where $\sigma_{\mathrm{cr}}$ is the cross-section for moderate lensing, $t$ is the cosmic time, and $\Phi_{\sigma}\left(z_{\mathrm{d}}, \sigma\right)$ is the lens velocity dispersion function (e.g. Turner, Ostriker \& Gott 1984, Gott, Park \& Lee 1989, Fukugita, Futamase \& Kasai 1990).

The lens velocity dispersion function is modelled by a modified Schechter function (Choi et al. 2007):

$\Phi_{\sigma}(\sigma) d \sigma=\Phi_{\sigma}^{\star}\left(\frac{\sigma}{\sigma_{\star}}\right)^{\alpha_{\sigma}} \exp \left[-\left(\frac{\sigma}{\sigma_{\star}}\right)^{\beta_{\sigma}}\right] \frac{\beta_{\sigma}}{\Gamma\left(\alpha_{\sigma} / \beta_{\sigma}\right)} \frac{d \sigma}{\sigma}$,

where $\Phi_{\sigma}^{\star}=8.0 \times 10^{-3} h^{3} \mathrm{Mpc}^{-3}, \alpha_{\sigma}=2.32 \pm 0.10, \beta_{\sigma}=2.67 \pm 0.07$, and $\sigma_{\star}=161 \pm 5 \mathrm{kms}^{-1}$. We limit the velocity dispersion to the interval $70 \sim 400 \mathrm{kms}^{-1}$ (Loeb \& Peebles 2003). Most massive earlytype galaxies were already assembled at $z<1$; beyond this redshift, the density of early-type galaxies declines significantly (e.g., Renzini 2006), and so we truncate the redshift of early-type background galaxies at redshift 2. Below this truncation redshift, we assume a constant comoving number density of lenses, implying the physical volume density evolves as $\Phi_{G, L}\left(z_{d}, \sigma\right)=\Phi_{G, L}(\sigma)(1+z)^{3}$, where the factor $(1+z)^{3}$ accounts for the expansion of the universe. Since most of the foreground (lensing) galaxies are below redshift 1 , this assumption will not have much effects on our results.

\subsection{Elliptical galaxy luminosity function}

The luminosity function (LF) of early-type galaxies is modelled by the Schechter (1976) form

$\Phi_{L}(L) d L=\Phi_{L}^{\star}\left(\frac{L}{L_{\star}}\right)^{\alpha_{L}} \exp \left(-\frac{L}{L_{\star}}\right) \frac{d L}{L_{\star}}$.

We adopt values from Choi et al. (2007) that were obtained using a galaxy sub-sample of the SDSS Data Release 5 with $M_{\star}-$ $5 \log _{10} h=-20.23 \pm 0.04, \alpha_{L}=-0.527 \pm 0.043$, and $\Phi_{L}^{\star}=$ $0.71 \times 10^{-2} h^{-3} \mathrm{Mpc}^{-3}$, appropriate for the SDSS $r$-band.

It is more convenient for our calculation here to use the luminosity function with absolute magnitude $M$ rather than $L$. Eq. 6 can be rewritten as

$\Phi_{L}(M) d M=0.4 \ln 10 \times \Phi_{L}^{\star} 10^{-0.4\left(M-M_{\star}\right)\left(\alpha_{L}+1\right)} \exp \left(-10^{-0.4\left(M-M_{\star}\right)}\right) d M,(7)$

where $M_{\star}$ is given below eq. 6 .

Each survey has its own flux limit which corresponds to a magnitude limit in a photometric band (e.g. the SDSS $r$ band), denoted by $m_{r}$. The absolute limiting magnitude, $M_{r}$, of a galaxy at redshift $z$ and galactic coordinates $(l, b)$, can be constructed from the apparent magnitude limit $m_{r}$ as follows:

$M_{r}=m_{r}-D M(z)-K_{r}(z)$,

where $D M(z)$ is the distance modulus, and $K_{r}(z)$ is the K-correction (Hogg 1999). Here we use the $K_{r}(z)$ given by Choi et al. (2007), and we have ignored dust extinction by the Milky Way and the foreground galaxy.

The lensing probability averaged over all source redshifts is given by (e.g., Wyithe et al.2010)

$$
<\tau>=\frac{1}{N_{\mathrm{G}}} \sum_{i=1}^{N_{\mathrm{G}}} \tau\left(z_{i}\right)=\frac{1}{N_{\mathrm{G}}} \int_{0}^{z_{s, \star}} d z \frac{d V_{\mathrm{C}}}{d z} \Psi_{G, S}\left(M\left(z_{\mathrm{s}}\right), z_{\mathrm{s}}\right) \tau\left(z_{\mathrm{s}}\right),
$$

where $N_{\mathrm{G}}$ is the total number of background elliptical galaxies, $V_{\mathrm{C}}$ is the comoving volume, and $\Psi_{G, S}\left(M_{r}\left(z_{\mathrm{s}}\right), z_{\mathrm{s}}\right)$ is the comoving number density of elliptical galaxies brighter than $M_{r}\left(z_{\mathrm{s}}\right)$ at redshift $z_{\mathrm{s}}$. $N_{G}$ can be calculated as

$N_{G}=\int_{0}^{z_{s, \star}} d z \frac{d V_{\mathrm{C}}}{d z} \Psi_{G, S}\left(M_{r}\left(z_{\mathrm{s}}\right), z_{\mathrm{s}}\right)$.

and $\Psi_{G, S}\left(M_{r}\left(z_{\mathrm{s}}\right), z_{\mathrm{s}}\right)$ as

$\Psi_{G, S}\left(M_{r}\left(z_{\mathrm{s}}\right), z_{\mathrm{s}}\right)=\int_{-\infty}^{M_{r}\left(z_{\mathrm{s}}\right)} \Phi_{\mathrm{L}}(M) d M$,

where $\Phi_{\mathrm{L}}(M)$ is the background elliptical galaxy luminosity function (see eq.7), and $M_{r}\left(z_{\mathrm{s}}\right)$ is the absolute limiting magnitude $M_{r}$ at redshift $z_{\mathrm{s}}$ corresponding to the magnitude limit $m_{r}$. To be conservative and for simplicity, we use the LSST single-visit depth limit $m_{r}=24.7$ in our optical depth calculation.

\subsection{Lens and source surface brightness profiles}

We model the background source (and the lens) as an elliptical galaxy with a de Vaucouleurs surface brightness profile: 
$I(R)=I_{\mathrm{e}} \exp \left(-7.67\left(\left(R / R_{\mathrm{e}}\right)^{1 / 4}-1\right)\right)$

where $R$ is the two-dimensional radius, $R_{\mathrm{e}}$ is the effective radius within which half of the light is enclosed and $I_{\mathrm{e}}$ is the surface brightness at the effective radius. We quote the effective radius as the geometric mean of the major and minor axes, $R_{\mathrm{e}}=\sqrt{a_{\mathrm{e}} b_{\mathrm{e}}}$. The total apparent magnitude $m_{\mathrm{T}}$, average magnitude within effective radius $\langle\mu\rangle_{\mathrm{e}}$ and effective radius $R_{\mathrm{e}}$ can be related by (Scodeggio et al. 1998,

$m_{\mathrm{T}}=-2.5 \log _{10}\left(\mathrm{~L}_{\mathrm{T}}\right)-48.6=\langle\mu\rangle_{\mathrm{e}}-1.9954-5 \log _{10}\left(\mathrm{R}_{\mathrm{e}}\right)$,

where $L_{\mathrm{T}}$ is the total flux of the elliptical galaxy and $R_{\mathrm{e}}$ is measured in $\operatorname{arcsec}$.

\subsection{Simulating images of early-type galaxies}

In an image of an early-type galaxy, in addition to the signal of the source, we also have the Poisson noise in the sky background, readout noise of the CCDs and the dark current. The signal-to-noise ratio is given by (McMaster et al. 2008)

$\frac{S}{N}=\frac{N_{\mathrm{S}} t}{\sqrt{N_{\mathrm{S}} t+n\left(N_{\mathrm{sky}} t+N_{\mathrm{DC}} t+N_{\mathrm{R}}^{2}\right)}}$,

where $N_{\mathrm{S}}$ is the number of photoelectrons from the source per unit time, $N_{\text {sky }}$ is the intensity of sky background in photoelectrons per pixel per unit time, $N_{\mathrm{DC}}$ is the dark current in electrons per pixel per unit time, $N_{\mathrm{R}}$ is the readout noise in electrons per pixel, $n$ is the number of pixels covered by an image of an early-type galaxy and $t$ is the exposure time.

To proceed further, we need to adopt a concrete case for the parameters in eq. 14. Here we restrict ourselves to LSST. The telescope will be sited in Cerro Pachón, Chile, with excellent median free-air seeing of $0 . " 7$ in the $r$ band. Correspondingly, the pixel scale is chosen to be $0 .{ }^{\prime \prime} 2$. The telescope will repeatedly survey the sky covering an area of 20000 square degrees with a cadence of $\approx 7$ days. The integration time $(t)$ for each individual frame is 15 seconds. We assume that the readout noise is the same for all exposures. For the Poisson noise in the sky background, readout noise of the CCDs and the dark current, we use the mean values given by the LSST Exposure Time Calculator ${ }^{3}$. Using these numbers, we verified that the photon numbers we obtain differ from those given by the LSST Exposure Time Calculator by less than 3\%.

Fig. 1. shows an example of a simulated moderately lensed background elliptical galaxy over-plotted with the best-fit ellipses from the IRAF task ELLIPSE (for more details, see \$3.2). Notice that we have not convolved the image with seeing.

To simulate a population of early-type galaxies, we use the method given in Appendix A of Bernardi et al. (2003a). Briefly, each galaxy is randomly assigned an absolute magnitude, $M_{\mathrm{r}}$, effective radius $R_{\mathrm{e}}$ and velocity dispersion $\sigma$ ( $V$ in the notation of Bernardi et al. 2003a), appropriate at redshift $z=0$. This procedure ensures the generated galaxies satisfy the observed correlations among observed properties, including the fundamental plane as given by Bernardi et al. (2003b)

$\log _{10} R_{\mathrm{e}}=\log _{10} \sigma+0.2\left(\langle\mu\rangle_{\mathrm{e}}-20.09\right)$.

We assign a typical error of $0.03 \mathrm{dex}$ in $\log \sigma$ and $0.02 \mathrm{dex}$ in $\log _{10} R_{\mathrm{e}}$. The scatter in the fundamental plane is quite small, on the order of $\approx 0.08$, and may be mostly due to measurement errors.

${ }^{3}$ http://dls.physics.ucdavis.edu:8080/etc4_3work/servlets/LsstEtc.html

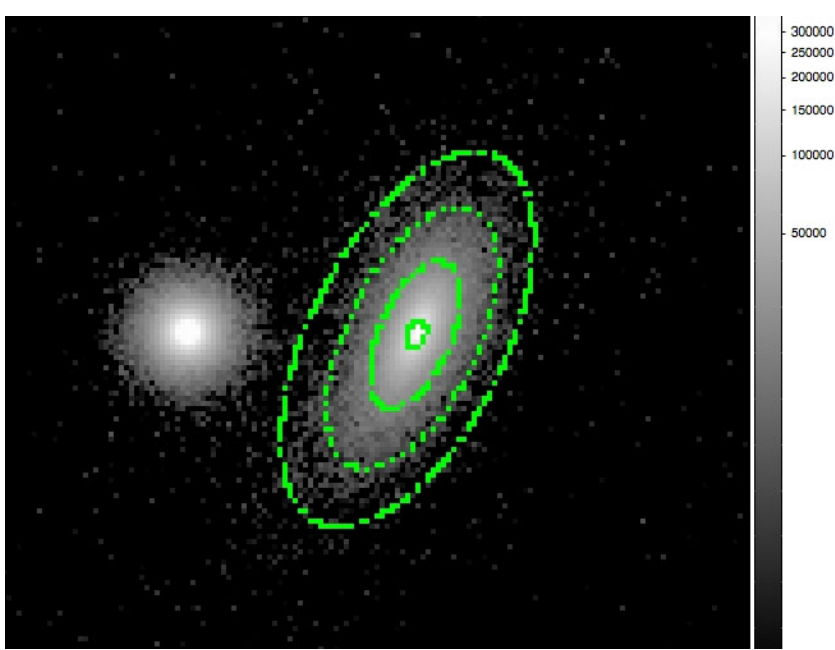

Figure 1. A simulated lensed image of a background elliptical galaxy, with a scale of $0 .{ }^{\prime \prime} 2$ per pixel. The foreground lens is at redshift 0.3 and the background elliptical galaxy (on the right) is at redshift 0.6 . The size of the image is $30 . " 0$ by $25 . " 6$. The distance between the two galaxy centres is 8.2 arcsec. The ellipses are obtained by running the IRAF task ELLIPSE (see $\$ 3.2$. Notice the small changes in the position angle and twists in the isophotes.

The Kormendy (1977) relation between the surface brightness and effective radius is given by (Bernardi et al. 2003a as quoted in Oohama et al. 2009)

$\langle\mu\rangle_{\mathrm{e}}=2.04 \log _{10} R_{\mathrm{e}}+18.7$,

where $\langle\mu\rangle_{\mathrm{e}}$ is the mean surface brightness (in mag/arcsec ${ }^{2}$ ) within the effective radius $\left(R_{\mathrm{e}}\right.$, in units of $\left.\mathrm{kpc}\right)$. The scatter in this relation is substantially larger than that in the fundamental plane (see \$3.4).

For LSST, the mean redshift of the galaxy sample is about 0.8 (see the LSST science book for details). However, to be specific and somewhat conservative, we use a lens redshift of 0.3 and a source redshift of 0.6. In reality, galaxy pairs will have a redshift distribution, but we can in principle bin the data and study the quantities we are interested, and so this simplification does not change the results of the paper. Notice that for the optical depth calculation, we still assume the lenses and sources have a redshift distribution, as described in $\$ 2$

For each random galaxy generated for redshift 0 , we shift it to redshift $z$ by taking into account its evolution (Bernardi et al. 2003a)

$M_{r}(z)=M_{r}(z=0)-Q z$,

where $Q=0.85$ for the $r$ band.

We assume a total exposure time of 6000 seconds (i.e., 400 exposures of 15 seconds). We further select only galaxies which satisfy $R_{\mathrm{e}}>0 . .^{\prime \prime} 7$, as a way to crudely account for the effects of seeing. In total, we generate 11623 pairs of foreground and background galaxies which satisfy these conditions. The ellipticity of the background galaxy is randomly drawn from the axis ratio distribution as given in Choi et al. (2007) (see their Fig.13). The lensed background galaxy images are obtained using the lens equation. The following analyses are based on this sample, although we apply a further cut in the isophote shape analysis (see below). 


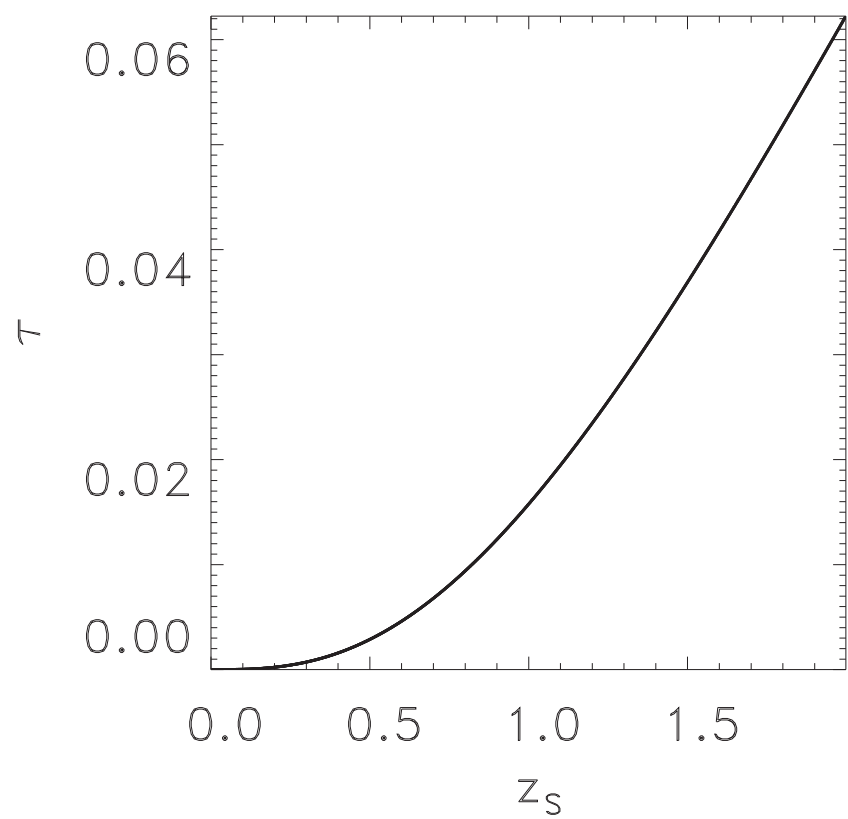

Figure 2. The optical depth, $\tau$, as a function of the redshift, $z_{\mathrm{s}}$. The crosssection for moderate lensing is taken as the area between $1 \theta_{\mathrm{E}}$ to $10 \theta_{\mathrm{E}}$.

\section{RESULTS}

\subsection{Optical depth}

In Fig. 2 we present the optical depth as a function of redshift. In this exercise, we take the moderate lensing cross-section for each galaxy as $\theta_{\mathrm{E}}$ to $10 \theta_{\mathrm{E}}$ (corresponding to $\mu=2$ and 1.1). Not surprisingly the optical depth increases with increasing redshift, reflecting the fact that the number of intervening galaxies increases for more distant background sources. The optical depth is about 0.0029 at $z=0.5,0.016$ at $z=1.0$, and 0.062 at $z=2.0$. The optical depth of averaged over all the background sources is about 0.0254 . This probability will scale linearly with the cross-section we adopt. For example, if the we take the cross-section from $2 \theta_{\mathrm{E}}$ to $5 \theta_{\mathrm{E}}$ (corresponding to $\mu=1.5$ and 1.2) then the probability will be reduced by a factor of roughly 5 to about 0.005 . The precise choice of crosssection will depend on the depth, seeing etc. of observations.

We have also calculated the magnification bias (Turner et al. 1984), which can be large for multiply-imaged quasars or galaxies. In our case, we find the magnification bias to be modest, only about 1.16. The magnification bias is small because the magnification in our case is by definition modest.

As we have mentioned in $\$ 2.5$ the redshift of the background sources is truncated at $z=2.0$. With this limitation, the surface number density of early-type galaxies is about 10 per square arcmin. For LSST, it will carry out a survey of $20000 \mathrm{deg}^{2}$ of the sky, and about 700 million elliptical galaxies will be detected. In Fig. 3 we present the distribution of the effective radius of the background elliptical galaxy sources. The number of elliptical galaxies drops steeply for larger effective radii. About $54.1 \%$ of the background sources have effective radii larger than $0 .{ }^{\prime \prime} 7$, the median free-air seeing at the site of LSST. Under this criterion, we estimate that there are about 9.5 million moderate galaxy lensing cases that will be observed by LSST to the single-visit depth $\left(m_{r}=24.7 \mathrm{mag}\right)$.

The predicted angular Einstein radius is shown in Fig. 4 As can be seen, the distribution peaks around 0.8 arcsec and has an extended tail out to larger separations. The distribution is similar to

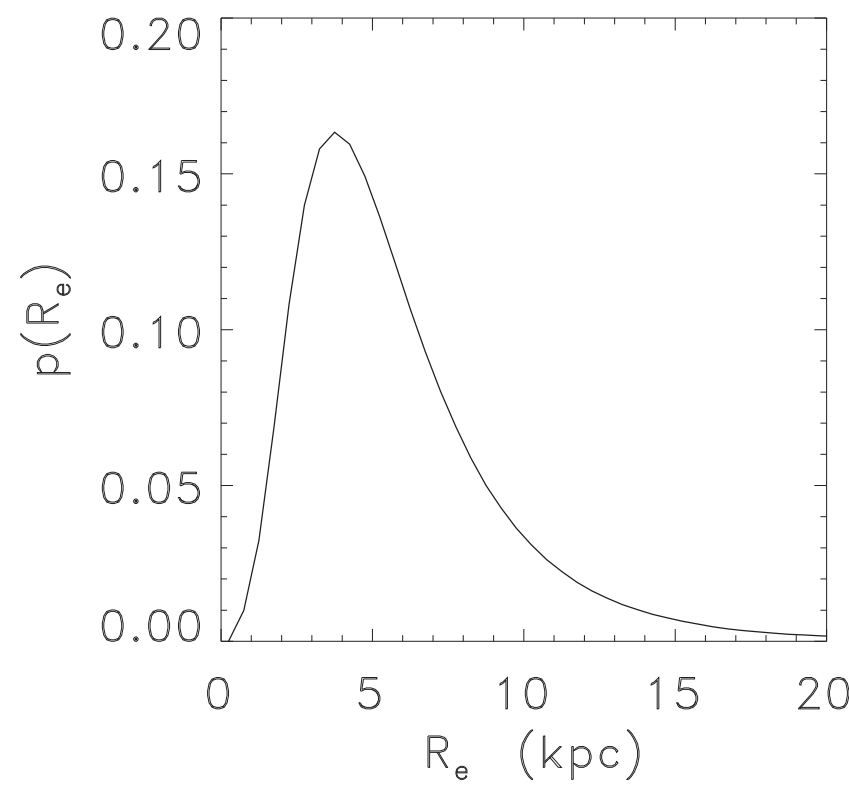

Figure 3. The probability density distribution of the effective radii of the background elliptical galaxies, all at redshift 0.6 . At this redshift, 1 arcsec corresponds to $4.7 h^{-1} \mathrm{kpc}$.

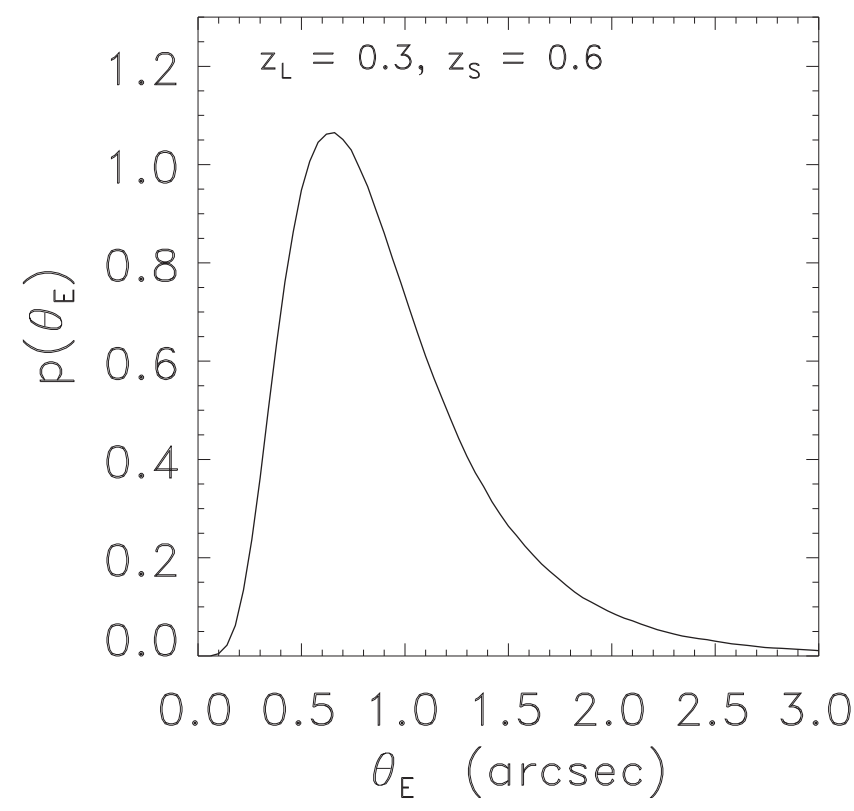

Figure 4. The probability density distribution of the angular Einstein radius $\theta_{\mathrm{E}}$ of the simulated lensing cases.

the observed one from the CLASS survey, even though the source and lens distributions there are somewhat different (see Fig. 11 in Browne et al. 2003).

\subsection{Isophotal distortions}

Surface photometry is performed on the simulated lensed images of the background elliptical galaxy by utilizing the IRAF task ELLIPSE (Jedrzejewski 1987). When running the ELLIPSE on the lensed images, the geometric centre, elllipticy and position angle are all allowed to vary freely, with a logarithmic step of 0.05 in the 

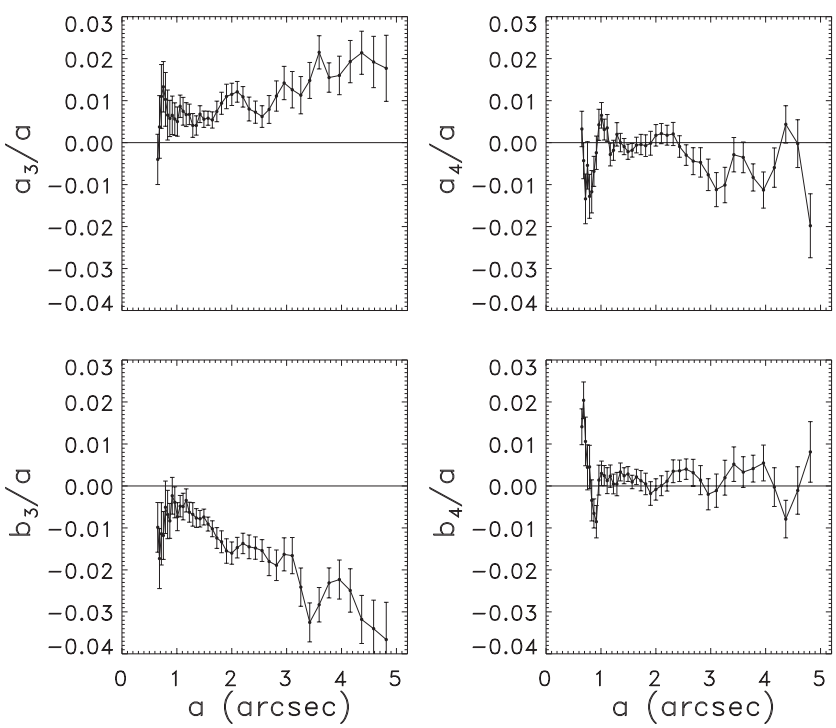

Figure 5. Coefficients of the Fourier series $a_{3} / a, a_{4} / a, b_{3} / a$ and $b_{4} / a$ obtained by the IRAF task ELLIPSE for the galaxy shown in Fig. 1] The horizontal line in each panel indicates the value for a perfect ellipse.

semi-major axis. The isophotes of the lensed images can be measured in different ways. Here we present the output of the task ELLIPSE. A detailed description of these measurements can be found in Hao et al. (2006), we only repeat the essentials here. The intensity along the ellipse is expanded in Fourier series

$I(\theta)=I_{0}+\sum\left(A_{n} \cos n \theta+B_{n} \sin n \theta\right)$

where $I_{0}$ is the intensity averaged over the ellipse, and $A_{n}$ and $B_{n}$ are the higher order Fourier coefficients. If an isophote is a perfect ellipse, then all the coefficients, $\left(A_{n}, B_{n}\right), n=1, \cdots, \infty$ will be exactly zero. We will use two quantities $a_{n}$ and $b_{n}$ extensively, which are related to the ELLIPSE outputs $A_{n}$ and $B_{n}$ by

$\frac{a_{n}}{a}=\frac{A_{n}}{\gamma a}$

where $\gamma=d I / d R$ is the local radial intensity gradient, and $a$ is the semi-major axis length.

For the galaxy shown in Fig. 1 the coefficients of the Fourier series $a_{3} / a, a_{4} / a, b_{3} / a$ and $b_{4} / a$ as a function of the semi-major axis radius are shown in Fig.5 The horizontal line in each panel is the value for a perfect ellipse. Notice that both $a_{3} / a$ and $b_{3} / a$ show significant deviations from the isophotes of a perfect ellipse.

For the same galaxy, we present the distribution of the apparent isophotal axis ratio $q$, position angle $\varphi$ in Fig.6. The horizontal line presents the ellipticity (position angle) before lensing. There are some small but systematic changes in these two quantities of a few percent for the example shown here. The $a_{4} / a$ and $b_{4} / a$ parameters describe whether an elliptical galaxy is disky or boxy, but we did not incorporate these into our image simulations. Instead we choose to focus on the $a_{3} / a$ and $b_{3} / a$ parameters.

In Fig 7 we present the distribution of the coefficients of the Fourier series $a_{3} / a, a_{4} / a, b_{3} / a$ and $b_{4} / a$ for cases where the separations between the foreground galaxy and background galaxy are between $\theta_{\mathrm{E}}+R_{\mathrm{e}, \mathrm{s}} \approx 2$. . $^{\prime} 0$ and 10 ." $^{\prime}$, where $R_{\mathrm{e}, \mathrm{s}}$ is the effective radius for the source. For each galaxy, these coefficients are the mean value within the region between $2 r_{s}$ and $1.5 R_{50}$, where $r_{s} \approx 0 .{ }^{\prime \prime} 7$ is the median seeing radius and $R_{50}$ is the Petrosian half-light radius. For SDSS, $R_{50}=0.71 R_{\mathrm{e}}$ for early-type galaxies described by the
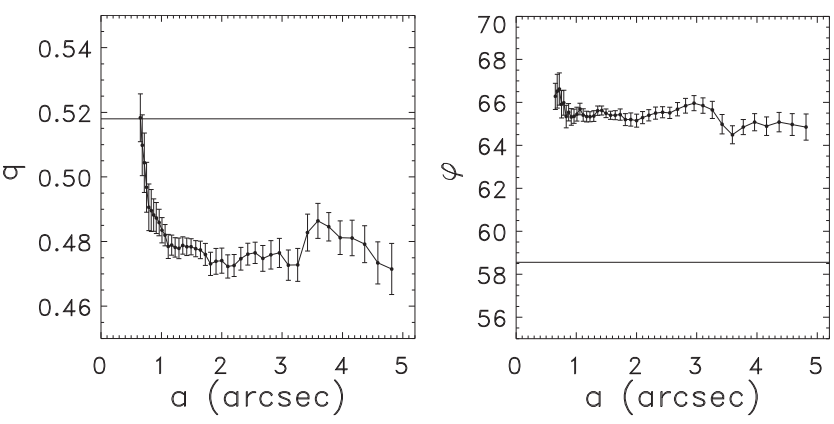

Figure 6. The probability density distributions of the apparent isophotal axis ratio $(q)$, position angle ( $\varphi$, in units of degrees) for the galaxy shown in Fig. 1

$R^{1 / 4}$ law (Hao et al. 2006, Graham et al. 2005). In the same plot, these distributions for separations between $2 .{ }^{\prime \prime} 0$ and $6 . " 0$ and between 2." 0 and 10." 0 are also shown. As expected, for background sources with smaller separations from the lens centre, the distributions of the coefficients are broader because the lensing effects are stronger in such cases. Notice that in the above analysis, we require $2 r_{s}>1.5 R_{50}$, this condition reduces the pair of galaxies from 11623 to 9094 .

Both the $a_{3} / a$ and $b_{3} / a$ distributions show large deviations from those of normal elliptical galaxies as studied by Hao et al. (2006). This is further illustrated in Fig. 8, here we plot the twodimensional distributions of these two quantities rather than as histograms from projection. We can see that the unlensed galaxies are mostly at the centre, while the lensed galaxies clearly show much larger scatters than the unlensed ones. Thus they can be used as effective indicators whether moderate gravitational lensing has occurred in a pair.

\subsection{Fundamental plane}

Gravitational lensing preserves the surface brightness of the source because of Liouville's theorem, but changes the apparent solid angle of a source. Following this, a natural expectation is that the fundamental plane of background elliptical sources should be affected by moderate gravitational lensing.

To obtain the lensed and unlensed effective radii and surface brightness, $R_{\mathrm{e}, \mathrm{L}}$ and $R_{\mathrm{e}}$, we fit the surface brightness profile of the lensed images of background elliptical galaxies with the $R^{1 / 4}$ law. In this model, we fix the centre of the galaxy, after which there are four remaining free parameters, $\left\{I_{\mathrm{e}}, R_{\mathrm{e}}, \varphi, q\right\}$. The $\chi^{2}$ function is given by

$\chi^{2}\left(I_{e}, R_{\mathrm{e}}, \varphi, q\right)=\sum_{i=1}^{N_{p}} \frac{\left(\mathcal{I}_{i}-\mathcal{I}_{i, 0}\right)^{2}}{\sigma_{i, 0}^{2}}$.

where $N_{\mathrm{p}}$ is the number of pixels of the lensed image. $\mathcal{I}_{i, 0}$ is the photon number in the $i^{\text {th }}$ pixel, $\sigma_{i, 0}$ is the Poisson noise $\sigma_{i, 0}=$ $\sqrt{I_{i, 0}}$. The degree of freedom is $N_{\text {dof }}=N_{\mathrm{p}}-4$. We use the routine gsl_multimin_fminimizer_nmsimplex in the GNU Scientific Library ${ }^{4}$ to find the $\chi^{2}$ minimum and obtain the effective radius; the mean surface brightness is then obtained by averaging the surface brightness within $R_{\mathrm{e}}$.

Fig. 9 shows the distribution of differences in effective radii

4 http://www.gnu.org/software/gsl/ 

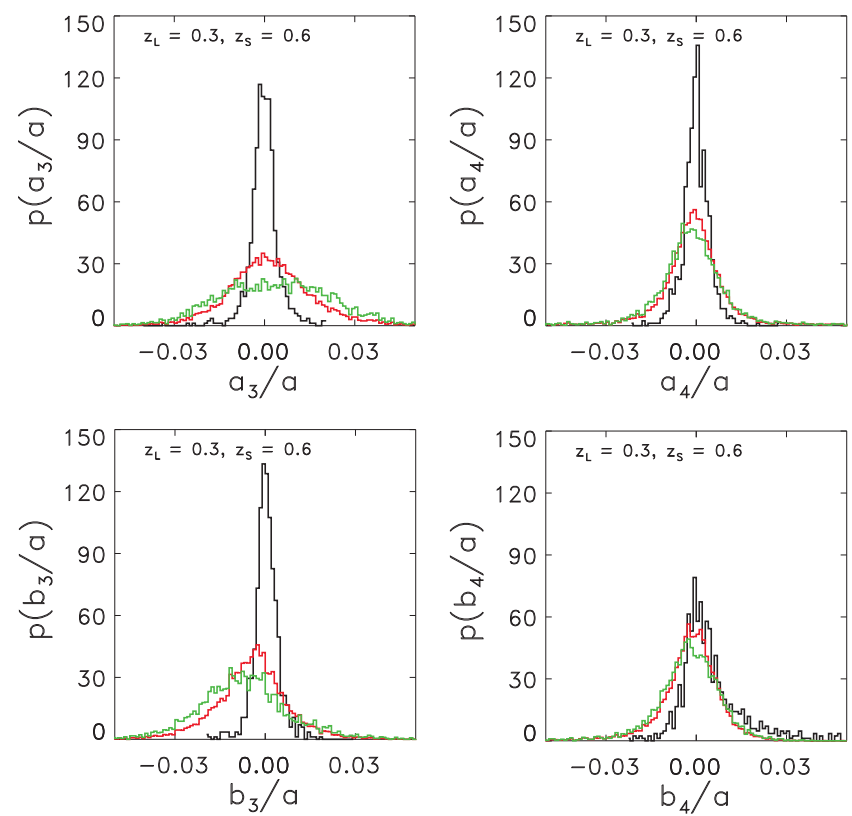

Figure 7. The probability density distributions of the coefficients of the Fourier series $a_{3} / a, a_{4} / a, b_{3} / a$ and $b_{4} / a$ obtained by the IRAF task ELLIPSE. The black solid histogram in each panel shows the distribution of the coefficients for a nearby sample of early-type galaxies from Hao et al. (2006). The green line is for moderately lensed galaxy pairs within separation between 2 to 6 arcsec while the red solid line is for galaxies from 2 to 10 arcsec.

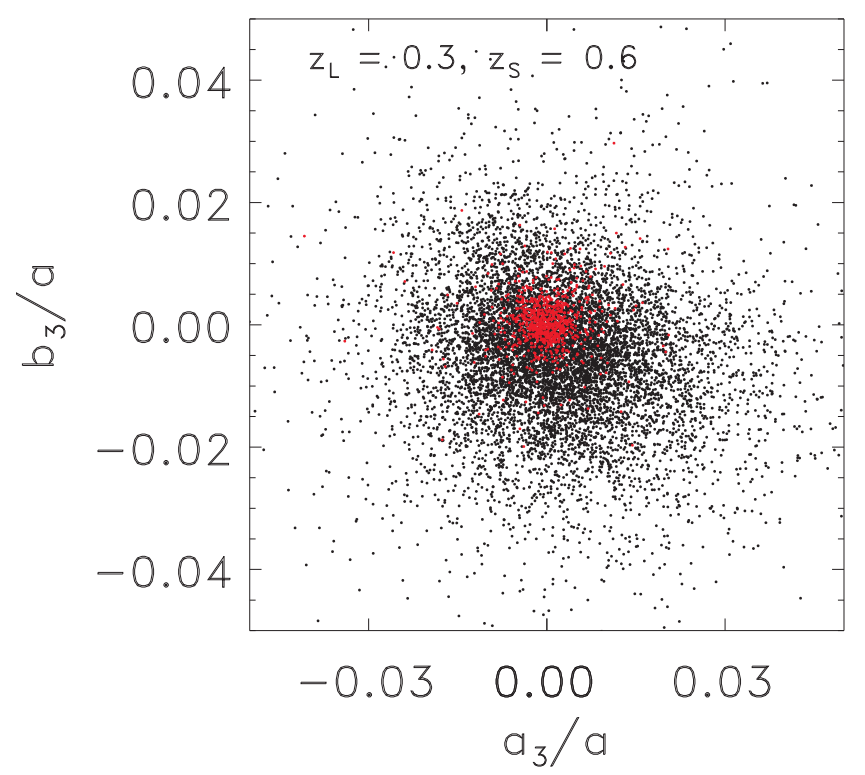

Figure 8. The scattered plot for the simulated galaxy pairs in the plane $a_{3} / a$ vs. $b_{3} / a$. The red dots are for unlensed galaxies while the black ones are for lensed ones. In total, there are 9094 pairs of galaxies.

between the lensed and unlensed sources, defined as $\Delta \log _{10} y R_{\mathrm{e}}=$ $\log _{10} R_{\mathrm{e}, \mathrm{L}}-\log _{10} R_{\mathrm{e}}$. The mean and median values of $\Delta \log _{10} \mathrm{R}_{\mathrm{e}}$ are 0.03 and 0.03 respectively. These values will depend on the separation range we take for the galaxy pair, here we have selected pairs with separations between $\sim 2$ to 10 arcsec.

Similarly, Fig. 10 shows the distribution of differences in the

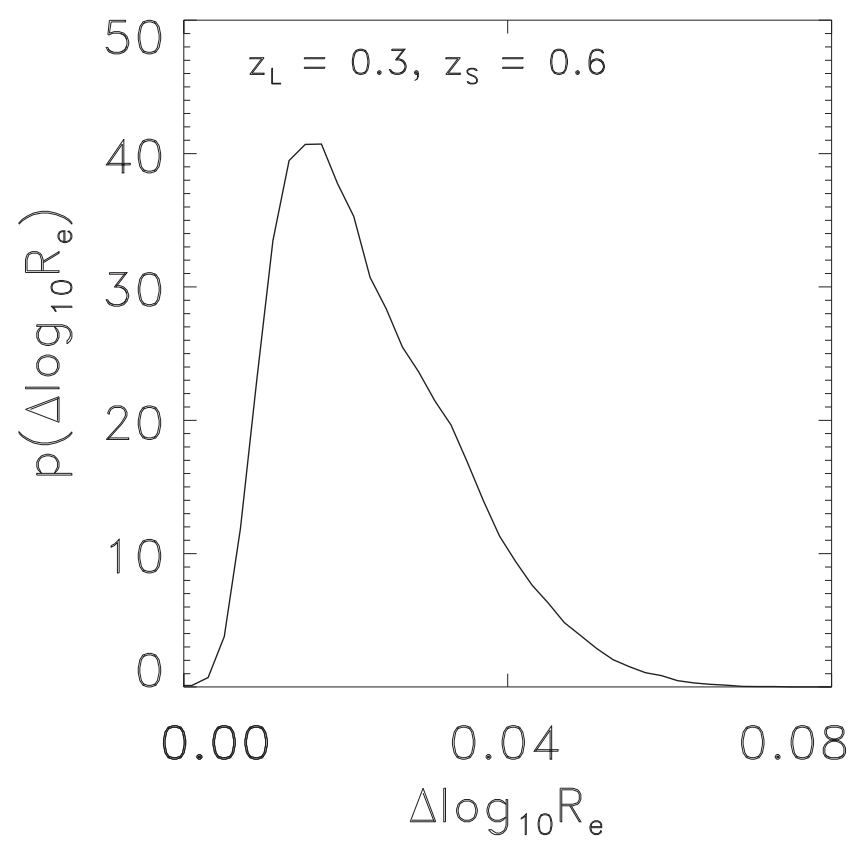

Figure 9. The probability density distribution of differences in effective radii between the lensed and unlensed sources in our simulated sample. Notice the systematic shift to a larger effective radius.

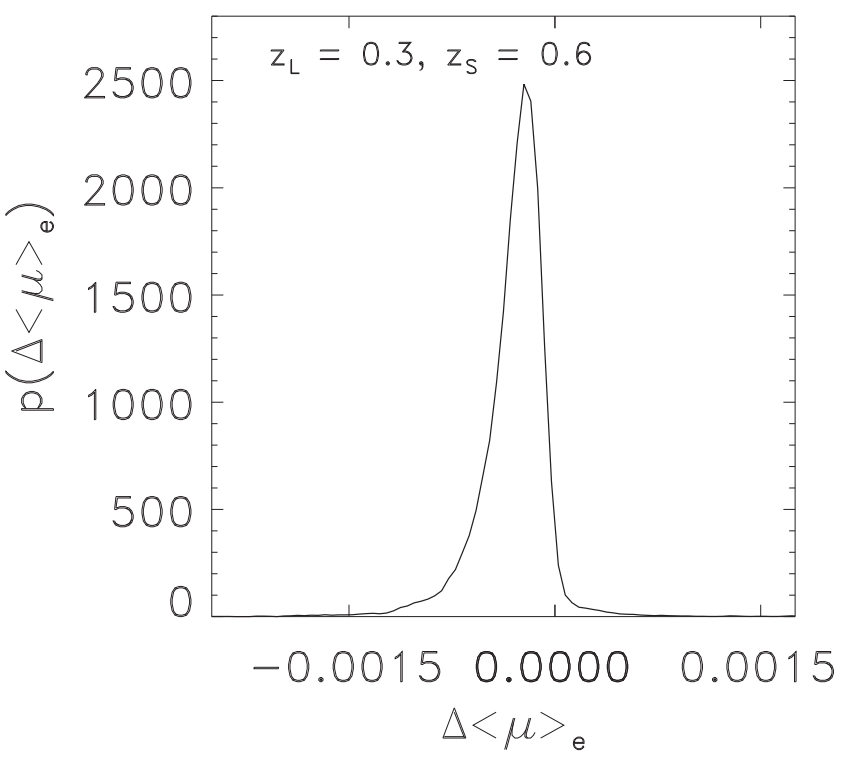

Figure 10. The probability density distribution of differences in mean surface brightness within effective radius between the lensed and unlensed sources in our simulated sample.

mean surface brightnesses within the effective radius between the lensed and unlensed sources, defined as $\Delta\langle\mu\rangle_{\mathrm{e}}=\langle\mu\rangle_{\mathrm{e}, \mathrm{L}}-\langle\mu\rangle_{\mathrm{e}}$, where $\langle\mu\rangle_{\mathrm{e}, \mathrm{L}}$ and $\langle\mu\rangle_{\mathrm{e}}$ are the mean surface brightness within the lensed and unlensed effective radius of the lensed images. The mean and median differences are -0.0003 and -0.0003 , which are much smaller than those for the effective radius, i.e., gravitational lensing does not affect the mean surface brightness within the effective radius.

The fundamental plane of the lensed elliptical galaxies is presented in Fig. 11 The black solid line shows the fundamental plane 


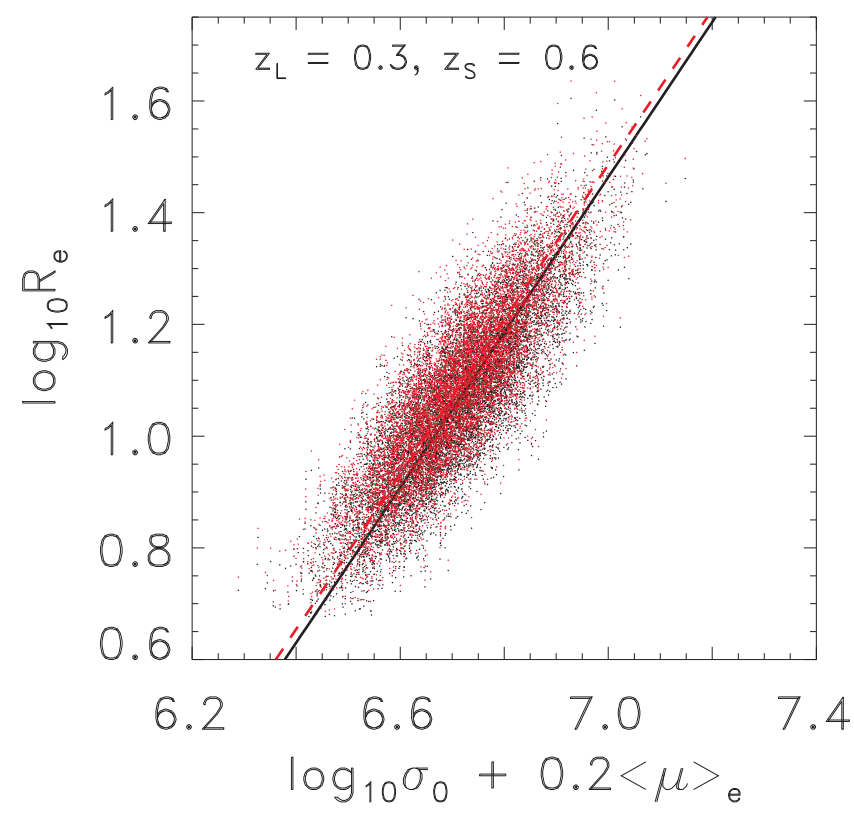

Figure 11. Fundamental plane of the lensed elliptical galaxies. The black solid line shows the fundamental plane for the background elliptical galaxies. The red dots are for the lensed elliptical galaxies with the best regression line shows as the red dashed line. The lens galaxies are at redshift $z_{1}=0.3$ and the source galaxies at $z_{\mathrm{s}}=0.6$.

used to produce the background elliptical galaxies (black dots) while the red symbols present the lensed elliptical galaxies. The best regression line for the lensed sample is shown as the dashed red line. On average an upward offset of about 0.03 is introduced by gravitational lensing.

\subsection{Kormendy relation}

To derive the systematic shifts in the fundamental plane, in principle we need to measure the velocity dispersion from spectroscopic observations. This will be very time consuming given the large number of early-type galaxies available from LSST. However, it is easier to measure the Kormendy (1977) relation between the surface brightness and effective radius, which can be obtained from imaging data and an approximate photometric redshift. In Fig. 12 we show the Kormendy relation, of the lensed and unlensed elliptical galaxies. On average, an upward offset of about 0.03 in the Kormendy Relation is seen in Fig. 11 As might be expected, this shift is similar to that in the fundamental plane. This offset is much smaller than the scatter orthogonal to the Kormendy relation (about 0.166 ) and the scatter in the direction of $\log R_{\mathrm{e}}$ (about 0.182).

\section{CONCLUSION AND DISCUSSION}

In this paper we have investigated the moderate lensing of background elliptical galaxies by intervening elliptical galaxies. We find the the optical depth for moderate lensing is on the order of $\sim 1 \%$. We have also performed image simulations based on the design specifications of LSST and obtained realistic lensed images of the background elliptical galaxies. The distortions of the lensed images have been quantified with the IRAF task ELLIPSE. We find moderately lensed galaxies can be potentially differentiated from normal galaxies as outliers in the coefficients of $a_{3} / a, b_{3} / a$ etc.

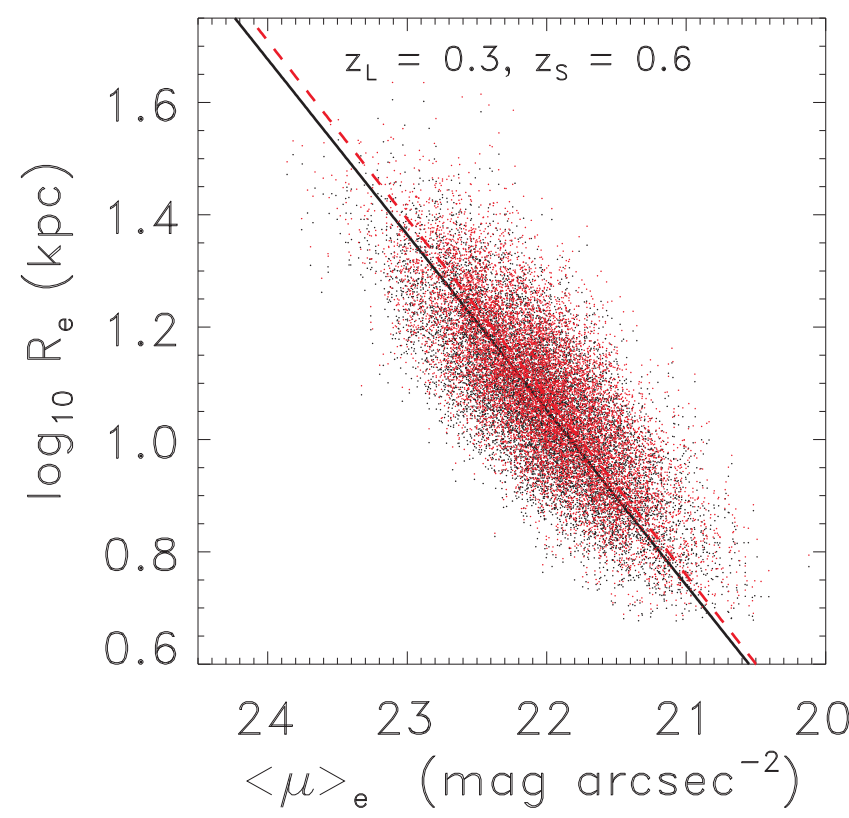

Figure 12. Kormendy relation of the elliptical galaxies. The black dashed line shows the Kormendy relation of the unlensed background elliptical galaxies (black dots). The red dashed line presents the relation of the lensed elliptical galaxies (red dots). In this figure, the lens galaxies are at redshift $z=0.3$ and the source galaxies at $z=0.6$.

We also explore the offsets in the Kormendy relation and fundamental plane of the lensed elliptical galaxies, and attribute these to the magnification effect of the effective radii, while the mean surface brightnesses within the radii are nearly unaffected. The systematic offsets are on the order of 0.03 in $\log R_{\mathrm{e}}$. To observe the shift in the fundamental plane, we need to determine the velocity dispersion spectroscopically while for the Kormendy relation, we only need the source redshift (e.g., from photometric redshift) to obtain the physical effective radius, so the observational demand is somewhat lower.

For strong lensing, it is possible to model an individual lens to extract information about the lens. For moderate lensing, this will be difficult, and its application will be mostly statistical. Since the scatter in the fundamental plane is small ( 0.08 in $\left.\log _{10} R_{\mathrm{e}}\right)$, a 0.03 shift can in principle be deteced with a few thousand galaxies. For the Monte Carlo simulations we discussed in $\$ 2$ we first fit the unlensed sample with a linear model and then renormalise the error bars so that the total $\chi^{2}$ per degree of freedom is unity. We then fit the same model to the lensed sample. If there were no systematic shifts, then the resulting $\chi^{2}$ should follow roughly a normal distribution with mean equal to $\chi_{\text {mean }}^{2}=N_{\text {dof }}=N-2$ and a dispersion of $\sigma_{\chi}=\sqrt{2 N_{\text {dof }}}$, where $N$ is the number of pairs of galaxies. Any significant deviation will indicate the no offset model is unsatisfactory. For our simulated sample, we find the $\chi^{2}$ for the no offset model is significantly higher than this expectation, and the excess $\chi^{2}$ above $\chi_{\text {mean }}^{2}$ is on the order of $4.2 \sigma_{\chi}$ and $6.6 \sigma_{\chi}$ for 1600 and 5000 pairs of galaxies. For the Kormendy relation, the corresponding significance levels are $3.4 \sigma_{\text {chi }}$ and $6.3 \sigma_{\text {chi }}$, somewhat lower than that in the fundamental plane, which is not surprising given the larger scatters in this relation.

In practice, there will be several complications since the lens and source redshifts may only be available photometrically. However, if there are no systematic errors, we can in principle bin the 
background galaxies, and stack them to find the systematic offset in the fundamental plane and Kormendy relation as a function of separation, which in turn provides strong constraints on the average profiles of galaxies at large radii. This complements the weak galaxy-galaxy method using shear.

At redshift 0.3 , the median Einstein radius is around 0.83 arcsec (corresponding to about $2.6 h^{-1} \mathrm{kpc}$ ), and moderate lensing can probe to $\sim 5 \theta_{E}$, about $13.2 h^{-1} \mathrm{kpc}$. This is an interesting radius, since it may be close to the regime where the density slope may be changing from isothermal $\left(\rho \propto r^{-2}\right.$, Koopmans 2009) to steeper profiles $\left(r^{-3}\right)$ : for a galactic-sized halo with virial radius of $r_{v}=200$ $\mathrm{kpc}$ and a concentration parameter of $c=10$, the radius where the density slope changes may be around $r_{v} / c=20 \mathrm{kpc}$.

Although the results presented here are promising, there are several points which need to be investigated further in the future. First, we do not fully consider the effects of seeing, as would be necessary in more realistic simulations. However, some of the quantities that we use, such as the Fourier components (see Fig.7), are already averaged over a range of radii and so the impact will be somewhat limited. Second, while many of the elliptical galaxies can be described well by the de Vaucouleurs profile, others are better described by the more general Sérsic (1968) profile. Third, for simplicity we adopt the singular isothermal lens model. In reality, several other models can better describe the lens galaxies. These include the singular isothermal ellipsoid model (Keeton \& Kochanek 1998), and the GNFW model (Zhao 1996, Chae 2002) which may be particularly suitable for studying the transition in the density profiles. In more detailed studies we could parameterise the foreground lens using these models and extract the best-fit parameters with more rigourous statistical methods, such as maximum likelihood or Bayesian techniques. It may be particular interesting to explore what we can learn with photometric redshifts alone for moderate gravitational lensing using Monte Carlo simulations.

\section{ACKNOWLEDGMENTS}

We thank Drs. Cheng Li, Hu Zhan and Zuhui Fan for helpful discussions. JW and SM acknowledge the Chinese Academy of Sciences and NSFC (grants 10821061 and 11033003) for financial support. MCS acknowledges financial support from the Peking University One Hundred Talent Fund (985) and NSFC grants 11043005 and 11010022 (International Young Scientist).

\section{REFERENCES}

Barkana R. 1998, ApJ, 502, 531

Bernardi et al. 2003a, AJ, 125, 1849

Bernardi et al. 2003b, AJ, 125, 1866

Blanton M. R. et al., 2003, ApJ, 592, 819

Browne I. W. A., et al. 2003, MNRAS, 341, 13

Bullock J. S. et al., 2001, MNRAS, 321, 559

Chae, K.-H. 2002, ApJ, 568, 500

Choi Y. Y., Park C., \& Vogeley M. S., 2007, ApJ, 658, 884

Davis A. N., Huterer D. \& Krauss L. M., 2003, 344, 1029

Futamase, T., Hattori, M., \& Hamana, T. 1998, ApJ, 508, L47

Fukugita, M., Futamase, T., \& Kasai, M., 1990, MNRAS, 246, P24

Gott, J. R., Park, M.-G., \& Lee, H.M 1989, ApJ, 338, 1

Graham, A. W., Driver, S. P., Petrosian, V., Conselice, C. J., \&

Goto, T. 2005, ApJ, 130, 1535
Hao, C. N., Mao, S., Deng, Z. G., Xia, X. Y. \& Wu Hong 2006, MNRAS, 370, 1339

Hogg D. W. 1999, arXiv:9905116

Jedrzejewski R. I., 1987, MNRAS, 226, 747

Kayser R., Refsdal S., Stabell R., 1986, A\&A, 166, 36

Keeton C. R., Kochanek C. S., 1998, ApJ, 495, 157

Komatsu E. et al., 2009, ApJs, 180, 330

Koopmans, L. V. E., et al. 2009, ApJ, 703, L51

Kormendy J., 1977, ApJ, 218, 333

Li L. X., Ostriker J. P., 2002, ApJ, 566, 652

Loeb A., Peebles P. J. E., 2003, ApJ, 589, 29

McMaster, Biretta, et al. 2008, WFPC2 Instrument Handbook, Version 10.0 (Baltimore: STScI)

Navarro J. F., Frenk C. S. \& White S. D. M., 1996, ApJ, 462, 563

Oguri M., Taruya A., Suto Y., Turner E. L., 2002, ApJ, 568, 488

Oohama, N., Okamura, S., Fukugita, M., Yasuda, N., \& Nakamura, O. 2009, ApJ, 705, 245

Press, W. H., Teukolsky, S. A., Vetterling, W. T., \& Flannery, B. P. 1992, Cambridge: University Press, —c1992, 2nd ed.,

Renzini A., 2006, ARA\&A, 44, 141

Schechter P., 1976, ApJ, 203, 297

Schneider P., Ehlers J., Falco E. E., 1992, Gravitational Lenses (Springer Verlag, Berlin)

Scodeggio M., Gavazzi G., Belsole E., Pierini D., Boselli A., 1998, MNRAS, 301, 1001

Sheth et al., 2003, ApJ, 594, 225

Sonnenfeld, A., Bertin, G., \& Lombardi, M. 2011, arXiv:1106.1442

Turner E. L., 1980, ApJ, 242, L135

Turner E. L., Ostriker J. P., \& Gott J. R., III. 1984, ApJ, 284, 1

Schneider, P., Kochanek, C. S., \& Wambsganss, J. 2006, Gravitational Lensing: Strong, Weak and Micro: , Saas-Fee Advanced Courses, Volume 33. ISBN 978-3-540-30309-1. Springer-Verlag (Berlin)

Seitz S., Saglia R. P., Bender R., Hopp U., Belloni P., Ziegler B., 1998, MNRAS, 298, 945

Sersic, J.-L. 1968, Atlas de Galaxias Australes (Co rdoba: Obs. Astron.)

Williams L. L. R., Lewis G. F., 1998, MNRAS, 294, 299

Wyithe J. S. B., Oh S. P., Pindor B., 2010, arXiv:1004.2081v1

Wyithe J. S. B., Winn J. N., Rusin D., 2003, ApJ, 583, 58

Wyithe J. S. B., Turner E. L., Spergel D. N., 2001, ApJ, 555, 504

Yee, H. K. C., Ellingson, E., Bechtold, J., Carlberg, R. G., \& Cuillandre, J.-C. 1996, AJ, 111, 1783

Zhao H. S., 1996, MNRAS, 278, 488 